\title{
Estudio comparativo de parámetros composicionales y nutricionales en leche de vaca, cabra y búfala, Antioquia, Colombia
}

\section{Comparative study of compositional and nutritional parameters in cow, goat and buffalo milk, Antioquia, Colombia}

\author{
OCAMPO G. RICARDO ${ }^{1 *}$ M.Sc, GOMEZ A. CATERINE ${ }^{1}$ MVZ, \\ RESTREPO V. DIEGO ${ }^{1}$ MVZ, CARDONA C. HENRY ${ }^{1}$ Ph.D.
}

${ }^{1}$ Universidad de Antioquia, Grupo de Investigación en Genética, Mejoramiento y Modelación Animal, GaMMA, Medellín, Colombia.

\section{Keywords:}

Minerals, fatty acid profile, Ruminants, Health.
Palabras Clave:

Minerales, perfil de ácidos grasos, rumiantes, salud.

\begin{abstract}
Milk is a fluid secreted by females of all species of mammals, which is used mainly to supply the nutritional needs of the newborn calf. It is an important source of carbohydrates, lipids, proteins, vitamins and minerals. Throughout history many countries have characterized the composition and nutritional quality of many mammals species milk, especially those that have been domesticated. However in Colombia there is insufficient information with respect to milk produced by different species to the cow. The aim of this study was to determine the composition and evaluate the nutritional quality of cow, goat and buffalo milk. Buffalo milk showed higher values in for parameters such as protein $(4,07 \%)$, fat $(7,23 \%)$, lactose $(5,06 \%)$, total solids $(16,81 \%)$ and solids non-fat $(9,91 \%)$ than goat and cow milk, making it an excellent alternative for the production and processing of milk products. On the other hand goat milk had the highest content of polyunsaturated fat $(0.1298 \mathrm{~g} / 100 \mathrm{~g})$ compared to cow milk and buffalo milk, which makes it more digestible and healthy for human consumption. All this led to the conclusion that each of these milk types has particular characteristics that make them more or less digestible and suitable for the manufacture of dairy products, which determines the advantages and disadvantages of each of the species.
\end{abstract}

\section{Resumen}

La leche es un líquido secretado por las hembras de todas las especies de mamíferos, la cual es utilizada principalmente para satisfacer las necesidades nutricionales de la cría recién nacida. Es una fuente importante de carbohidratos, lípidos, proteínas, vitaminas y minerales. A lo largo de la historia en varios países se ha caracterizado la composición y calidad nutricional de la leche de numerosas especies de mamíferos, especialmente las que han sido domesticadas. Sin embargo en Colombia no hay suficiente información en lo que respecta a leches producidas por especies no vacunas. El objetivo del presente estudio fue determinar la composición y evaluar la calidad nutricional de las leches de vaca, cabra y búfalo. La leche de búfala presento valores más altos para parámetros tales como la proteína $(4,07 \%)$, grasa $(7,23 \%)$, lactosa $(5,06 \%)$, solidos totales $(16,81 \%)$ y solidos no grasos $(9,91 \%)$ que las leches de cabra y vaca, lo cual la convierte en una excelente alternativa para la producción y transformación de productos. De otro lado la leche de cabra presento el mayor contenido de grasa poliinsaturada $(0,1298 \mathrm{~g} / 100 \mathrm{~g})$ comparadas con las leches de vaca y búfala, lo que la hace más digestible y saludable para el consumo humano. Todo lo anterior llevó a la conclusión de que cada uno de estos tipos de leche tiene características particulares que las hacen más o menos digestibles y adecuadas para la fabricación de productos lácteos, lo cual determina las ventajas y desventajas de cada una de las especies. 


\section{Introducción}

Dentro de la producción de alimentos de origen animal, la leche, es sin duda, la que presenta un volumen de producción y consumo más elevado, seguida de la carne y posteriormente de los huevos, debido en gran parte a que es una importante fuente de todos los nutrientes básicos requeridos por los mamíferos (incluyendo a los seres humanos) (KANWAL et al., 2004).

La leche es un líquido secretado por las glándulas mamaria de las hembras de los mamíferos, tras el nacimiento de la cría. Se trata de un líquido de composición compleja, blanco y opaco, de sabor dulce y reacción iónica $(\mathrm{pH})$ aproximadamente neutro (BALLARD y MORROW, 2013). La función natural de la leche es la de ser el alimento exclusivo de los mamíferos jóvenes durante el periodo crítico de su existencia tras el nacimiento, cuando el desarrollo es rápido y no puede ser sustituida por otros alimentos (CASHMAN, 2006).

Respecto a su composición, las diferentes especies de mamíferos producen leches que, en términos generales, tienen composición semejante pero pueden presentar diferencias importantes en su composición porcentual y tener como consecuencia, propiedades muy diferentes entre ellas (mayor o menor digestibilidad, mayores porcentajes de rendimiento en la elaboración de productos lácteos, etc.) (KANWAL et al., 2004).

Dentro de las especies las diferencias en la composición pueden estar influenciadas por varios factores tales como la raza, genética, alimentación y el medio ambiente que se le brinde al animal (MESTAWET et al., 2012). Es por esto que en varios países del mundo se han realizado numerosos estudios para establecer y evaluar la composición y calidad nutricional de la leche de sus animales en varias presentaciones; cruda, en polvo, etc. (ADLER et al., 2013; DIAN-BO ZHAO et al., 2015; PETIT, 2015; BARRUCAND y RAYNAL-LJUTOVAC, 2007; COUVREUR, 2006; MESTAWET et al., 2012).

La cadena de lácteos en el mundo ha tenido como fuente primaria principal la leche de algunos mamíferos pertenecientes a las especies bovina (vacunos y búfalos), caprina, ovina, etc. (FERNÁNDEZ et al., 2008). Sin embargo a escala mundial, la leche de vaca es la que posee mayores niveles de producción y consumo sobre las demás, y es así como según un estimado realizado por la FAO (2013), de los 769 millones de toneladas de leche producidas en el mundo para el año 2013, el 83\% corresponde a leche vacuna, seguida de la leche de búfala $(12,9 \%)$, de cabra $(2,3 \%)$ y de oveja $(1,3 \%)$.

Aunque la producción la leche de ganado vacuno supera ampliamente a las demás, los productos lácteos de los ganados bubalino y caprino pueden representar una alternativa rentable a los productores debido a sus características composicionales y nutricionales específicas, que las hacen aptas para la manufacturación de productos elaborados de muy alta calidad como los quesos y yogures gourmet (BALLARD y MORROW, 2013).

Sin embargo debido al desconocimiento generalizado de estas especies, los consumidores están solicitando información acerca de la calidad higiénica, composición y propiedades nutricionales de estos productos elaborados a partir de leche no vacuna para su aceptación y consumo, información que muchas veces es insuficiente o simplemente no está disponible para algunos países.

Aunque existen algunas publicaciones importantes sobre la composición y calidad nutricional de las leches de búfala y cabra en otros países (LEITNER et al., 2015; KONDYLI et al., 2012; VIEITEZ et al., 2016; ZOTOS et al., 2014; MESTAWET et al., 2012; REHMAN y SALARIA, 2005), es de resaltar que en Colombia no hay información relativa a moléculas específicas con propiedades nutricionales, como por ejemplo ácidos grasos y sus variantes, colesterol, vitaminas y minerales en general.

Si bien en el país hay un gran potencial para trabajar en la producción de leches de cabra y búfala, es necesario empezar a realizar estudios de este tipo y teniendo un mayor conocimiento de las propiedades nutricionales y composicionales de las leches de estas especies no vacunas se podrá fomentar cada día más su producción y consumo dentro de la población colombiana.

El objetivo de este estudio fue caracterizar y evaluar la calidad nutricional y composicional de la leche de vaca, cabra y búfalo en el departamento de Antioquia. 


\section{Materiales y métodos}

Se colectaron seis muestras de leche $(200 \mathrm{ml}$ cada una) en cuatro municipios del departamento de Antioquia; dos muestras de leche de vaca en el municipio de San pedro de los Milagros, dos muestras de leche de búfala en el municipio de Caucasia y dos muestras de leche de cabra en los municipios de Copacabana y la Ceja.

Cada una de las muestras fue tomada del tanque de enfriamiento, el cual fue previamente mezclado durante cinco minutos aproximadamente para homogenizar y así tomar la muestra. Todas las muestras de leche fueron tomadas en recipientes estériles y posterior a la toma cada una fue rotulada, refrigerada y transportada al laboratorio en el menor tiempo posible para garantizar la integridad de la muestra. En el laboratorio, para determinar la calidad nutricional y composicional de cada una de las muestras, varios análisis fueron realizados.

En el Laboratorio de Calidad e Inocuidad de la Leche ubicado en la Facultad de Ciencias Agrarias de la Universidad de Antioquia se evaluó el contenido de lactosa, proteína, grasa, sólidos totales, sólidos no grasos, nitrógeno ureico en leche por el método de infrarrojo utilizando el equipo MILKOSCAN ${ }^{\mathrm{TM}} \mathrm{FT}+$.

En el Laboratorio de Alimentación y Nutrición Humana de la Universidad de Antioquia se determinaron la concentración de ácidos grasos saturados, insaturados y poliinsaturados. Los lípidos de cada una de las muestras de leche fueron extraídos utilizando cloroformo metanol, según el método descrito por GUTIÉRREZ, RATTI y BELKACEMI (2008), con algunas modificaciones. Los ácidos grasos fueron analizados utilizando un cromatógrafo de gases Agilent 7890A (Agilent, Santa Clara, CA, USA), equipado con un detector de ionización de llama (FID). Las condiciones de análisis fueron las siguientes: Helio $(2,0 \mathrm{~mL} / \mathrm{min})$ fue utilizado como gas de arrastre; las temperaturas del inyector y detector se fijaron en $250^{\circ} \mathrm{C}$; la temperatura del horno fue inicialmente $60^{\circ} \mathrm{C}(1 \mathrm{~min})$ y luego se incrementó a $190^{\circ} \mathrm{C}$ a razón de $20^{\circ} \mathrm{C} / \mathrm{min}$, manteniéndose a esta última temperatura durante 12,5 min (GUTIERREZ et al., 2010). Las muestras fueron inyectadas en una columna capilar BPX-70 $(60 \mathrm{~m}$ x 0,25 mm x $0,25 \mu \mathrm{m}$; SGE, Melbourne, Australia) utilizando un split de 1:20. Los ácidos grasos fueron identificados por comparación de los tiempos de retención de los picos obtenidos con los de estándares certificados (FAME MIX C4-C24, CLA FAME, Sigma Aldrich, St. Louis, MO, USA). Los picos fueron integrados utilizando un software de captura de datos (Agilent ChemStation, versión B.04.01), y la cuantificación de los ácidos grasos se realizó por medio de calibración por estándar externo (GUTIERREZ et al., 2010).

En el Laboratorio Instrumental de la Universidad Nacional de Colombia (Sede Medellín) se midió la concentración de los minerales calcio $(\mathrm{Ca})$, fósforo $(\mathrm{P})$, magnesio $(\mathrm{Mg})$ y potasio $(\mathrm{K})$ en cada una de las muestras. Para la cuantificación de $\mathrm{Ca}, \mathrm{Mg}$ y $\mathrm{K}$ se utilizó espectrofotometría de absorción atómica (Basado en la Norma Técnica Colombiana 5151) y para P se utilizó espectrofotometría ultravioleta visible (Basado en Norma Técnica Colombiana 4981).

\section{Resultados}

El análisis de composicional de cada una de las leches de las especies analizadas se muestra en la Tabla 1.

Tabla 1. Análisis composicional de cada una de las muestras.

\begin{tabular}{|c|c|c|c|c|c|c|c|}
\hline Especie & Muestra & $\%$ lactosa & $\%$ grasa & \% proteína & $\% \mathrm{ST}$ & $\%$ SNG & MUN (mg/dl) \\
\hline \multirow{3}{*}{ Vaca } & 1 & 4,49 & 3,42 & 2,95 & 11,71 & 8,22 & 17,4 \\
\hline & 2 & 4,5 & 3,77 & 3,09 & 12,15 & 8,37 & 18,8 \\
\hline & Promedio & $4,50 \pm 0,01$ & $3,60 \pm 0,25$ & $3,02 \pm 0,1$ & $11,93 \pm 0,31$ & $8,30 \pm 0,11$ & $18,10 \pm 0,99$ \\
\hline \multirow{3}{*}{ Cabra } & 3 & 4,21 & 4,22 & 3,21 & 12,66 & 8,2 & 32,4 \\
\hline & 4 & 4,19 & 4,65 & 2,8 & 12,52 & 7,77 & 24,3 \\
\hline & Promedio & $4,20 \pm 0,01$ & $4,44 \pm 0,30$ & $3,01 \pm 0,29$ & $12,59 \pm 0,10$ & $7,99 \pm 0,30$ & $28,35 \pm 5,73$ \\
\hline \multirow{3}{*}{ Búfala } & 5 & 5,07 & 7,49 & 4,22 & 17,18 & 10,07 & 21,1 \\
\hline & 6 & 5,05 & 6,98 & 3,93 & 16,45 & 9,76 & 16,9 \\
\hline & Promedio & $5,06 \pm 0,01$ & $7,24 \pm 0,36$ & $4,08 \pm 0,21$ & $16,82 \pm 0,52$ & $9,92 \pm 0,22$ & $19 \pm 2,97$ \\
\hline
\end{tabular}


En términos generales, se puede observar que cuando se comparan la composición porcentual de lactosa, grasa, proteína, ST y SNG entre las muestras de leche de la misma especie no se observa diferencias notables entre las fincas muestreadas. Sin embargo, respecto a la concentración de nitrógeno ureico en leche (MUN), se puede observar que hay diferencia entre las mismas muestras de leche de cabra y búfala.

Cuando se comparó la composición porcentual entre las muestras de leche de las distintas especies, se puede observar que la leche de búfala posee una mayor concentración de lactosa, grasa, proteína, ST y SNG que la leche de vaca y cabra. Sin embargo la leche de cabra posee valores más altos de MUN cuando se compara con los otros tipos de leche.
Respecto a la composición y contenido de ácidos grasos saturados, insaturados y poliinsaturados en cada una de las muestras de leche analizadas, varias medidas se resumen en la Tabla 2.

De acuerdo a la Tabla 2, se puede observar que la leche de vaca posee un bajo contenido de ácidos grasos de cadena corta y media. En contraste la leche de cabra posee altos contenidos de estos tipos de ácidos, además de que también presenta altos contenidos de ácidos como el caprilico, caprico y laurico. De otro lado, la leche de búfala es la que posee mayores contenidos de ácidos grasos de cadena larga como el palmítico, esteárico entre otros, y en consecuencia, la leche de búfala es la que posee mayor contenido de grasa saturada, seguida por la de cabra y la de vaca, respectivamente.

Tabla 2. Análisis de ácidos grasos (g/100g).

\begin{tabular}{|c|c|c|c|c|c|c|c|c|c|}
\hline \multirow[b]{2}{*}{ Ácido graso } & \multicolumn{3}{|c|}{ VACA } & \multicolumn{3}{|c|}{ CABRA } & \multicolumn{3}{|c|}{ BÚFALA } \\
\hline & Muestra 1 & Muestra 2 & Promedio & Muestra 1 & Muestra 2 & Promedio & Muestra 1 & Muestra 2 & Promedio \\
\hline C4:0 ac. Butírico & 0,066 & 0,063 & $0,064 \pm 0,00$ & 0,053 & 0,070 & $0,061 \pm \mathbf{0 , 0 1}$ & 0,131 & 0,122 & $0,127 \pm \mathbf{0 , 0 1}$ \\
\hline C6:0 ac. Caproico & 0,041 & 0,039 & $0,040 \pm 0,00$ & 0,061 & 0,056 & $0,059 \pm 0,00$ & 0,064 & 0,056 & $0,060 \pm 0,01$ \\
\hline C8:0 ac. Caprílico & 0,025 & 0,024 & $0,025 \pm 0,00$ & 0,079 & 0,055 & $0,067 \pm 0,02$ & 0,032 & 0,026 & $0,029 \pm 0,00$ \\
\hline C10:0 ac. Capríco & 0,063 & 0,060 & $0,061 \pm 0,00$ & 0,315 & 0,183 & $0,249 \pm 0,09$ & 0,103 & 0,067 & $0,085 \pm \mathbf{0 , 0 3}$ \\
\hline C12:0 ac. Laurico & 0,075 & 0,082 & $0,078 \pm 0,00$ & 0,180 & 0,080 & $0,130 \pm 0,07$ & 0,104 & 0,087 & $0,095 \pm 0,01$ \\
\hline C13:0 ac. Tridecanoico & 0,003 & 0,003 & $0,003 \pm \mathbf{0 , 0 0}$ & 0,004 & 0,002 & $0,003 \pm 0,00$ & 0,006 & 0,006 & $0,006 \pm 0,00$ \\
\hline C14:0 ac. Miristico & 0,329 & 0,348 & $0,338 \pm 0,01$ & 0,413 & 0,267 & $0,340 \pm 0,10$ & 0,639 & 0,579 & $0,609 \pm 0,04$ \\
\hline C15:0 ac. Pentadecanoico & 0,040 & 0,047 & $0,044 \pm 0,00$ & 0,036 & 0,027 & $0,032 \pm 0,01$ & 0,110 & 0,101 & $0,106 \pm 0,01$ \\
\hline C16:0 ac. Palmítico & 0,906 & 1,057 & $0,981 \pm 0,11$ & 1,120 & 1,126 & $1,123 \pm 0,00$ & 2,211 & 2,104 & $2,158 \pm 0,08$ \\
\hline C17:0 ac. Heptadecanoico & 0,025 & 0,031 & $0,028 \pm 0,00$ & 0,019 & 0,024 & $0,021 \pm 0,00$ & 0,096 & 0,092 & $0,094 \pm \mathbf{0 , 0 0}$ \\
\hline C18:0 ac. Estearico & 0,549 & 0,617 & $0,583 \pm 0,05$ & 0,432 & 0,969 & $0,700 \pm 0,38$ & 1,238 & 1,135 & $1,187 \pm \mathbf{0 , 0 7}$ \\
\hline C20:0 ac. Araquidico & 0,007 & 0,008 & $0,008 \pm 0,00$ & 0,011 & 0,016 & $0,013 \pm 0,00$ & 0,020 & 0,018 & $0,019 \pm 0,00$ \\
\hline C22:0 ac. Behenico & 0,003 & 0,003 & $0,003 \pm 0,00$ & 0,002 & 0,004 & $0,003 \pm 0,00$ & 0,009 & 0,009 & $0,009 \pm 0,00$ \\
\hline C23:0 ac. Tricosanoico & ND & 0,003 & 0,003 & ND & ND & 0 & 0,008 & 0,008 & $0,008 \pm 0,00$ \\
\hline C24:0 ac. Lignocerico & ND & 0,002 & 0,002 & ND & ND & 0 & 0,009 & 0,009 & $0,009 \pm 0,00$ \\
\hline $\begin{array}{l}\text { TOTAL } \\
\text { GRASA SATURADA }\end{array}$ & 2,131 & 2,384 & $2,257 \pm 0,18$ & 2,724 & 2,879 & $2,802 \pm 0,11$ & 4,781 & 4,418 & $4,600 \pm 0,26$ \\
\hline C14:1 ac. Mirístico & 0,022 & 0,027 & $0,025 \pm 0,00$ & 0,007 & 0,002 & $0,004 \pm 0,00$ & 0,040 & 0,035 & $0,037 \pm 0,00$ \\
\hline C16:1 ac. Palmitoleico & 0,035 & 0,051 & $0,043 \pm 0,01$ & 0,030 & 0,024 & $0,027 \pm 0,00$ & 0,126 & 0,125 & $0,125 \pm 0,00$ \\
\hline C18:1n9c ac. Oleico & 0,859 & 1,051 & $0,955 \pm 0,14$ & 0,849 & 1,239 & $1,044 \pm 0,28$ & 1,864 & 1,896 & $1,880 \pm 0,02$ \\
\hline $\begin{array}{l}\text { C20: } 1 \mathrm{n} 9 \text { ac. } \\
\text { Cis-11-eicosenoico }\end{array}$ & ND & ND & 0,000 & 0,003 & 0,004 & $0,003 \pm 0,00$ & ND & ND & 0 \\
\hline $\begin{array}{l}\text { TOTAL GRASA } \\
\text { MONOINSATURADA }\end{array}$ & 0,916 & 1,129 & $1,022 \pm 0,15$ & 0,856 & 1,269 & $1,063 \pm 0,29$ & 2,030 & 2,056 & $2,043 \pm 0,02$ \\
\hline C18:2n6c ac. Linoleico & 0,056 & 0,035 & $0,046 \pm 0,01$ & 0,106 & 0,123 & $0,114 \pm \mathbf{0 , 0 1}$ & 0,038 & 0,041 & $0,040 \pm 0,00$ \\
\hline C18:3n3 ac. Linolenico & 0,010 & 0,013 & $0,012 \pm \mathbf{0 , 0 0}$ & 0,005 & 0,009 & $0,007 \pm 0,00$ & 0,027 & 0,028 & $0,027 \pm 0,00$ \\
\hline $\begin{array}{l}\text { C20:3n6 ac. } \\
\text { Cis-8,11,14-eicosatrienoico }\end{array}$ & 0,002 & ND & 0,002 & ND & ND & 0 & ND & ND & 0 \\
\hline $\mathrm{C} 20: 4 \mathrm{n} 6$ ac. Araquidonico & 0,004 & 0,003 & $0,003 \pm 0,00$ & 0,007 & 0,010 & $0,009 \pm 0,00$ & ND & ND & 0 \\
\hline $\begin{array}{l}\text { TOTAL GRASA } \\
\text { POLIINSATURADA }\end{array}$ & 0,072 & 0,052 & $0,062 \pm 0,01$ & 0,118 & 0,142 & $0,130 \pm 0,02$ & 0,065 & 0,069 & $0,067 \pm 0,00$ \\
\hline GRASA TOTAL & 3,281 & 3,747 & $3,514 \pm 0,33$ & 3,934 & 4,510 & $4,222 \pm 0,41$ & 7,226 & 6,874 & $7,050 \pm 0,25$ \\
\hline
\end{tabular}


Respecto a la grasa monoinsaturada, nuevamente la leche de búfala es la que posee mayor cantidad, seguido por la leche de cabra y vaca. Hay que resaltar que en la leche de cabra fue la única en la que se detectó el ácido graso Cis-11-eicosenoico.

En cuanto al contenido de grasa poliinsaturada, la leche de cabra es la que posee la mayor cantidad, seguida por la leche de búfala y la de vaca. Sin embargo la leche de vaca es la que posee mayor contenido de ácido araquidónico además que fue la única especie en la que se detectó el ácido eicosatrienoico.

En el análisis del contenido de los minerales $\mathrm{Ca}, \mathrm{P}$, $\mathrm{K}$ y Mg en cada una de las muestras analizadas se puede observar que entre las muestras de leche correspondientes a la misma especie se observan algunas diferencias en cuanto al contenido del mineral analizado. Cuando se compara el contenido de minerales en las leches de las diferentes especies, se puede observar que la leche de búfala posee mayores contenidos de $\mathrm{Ca}$, $\mathrm{P}$ y Mg que las leches de cabra y vaca (Tabla 3 ). De otro lado, el $\mathrm{K}$ se presenta en mayor cantidad en la leche vaca, seguido de cerca por la leche de cabra y en menor contenido en la leche de búfala.

\section{Discusión}

La leche de vaca es la materia prima más universal para el procesamiento de una amplia gama de productos manufacturados utilizados en la alimentación humana dado el alto contenido de grasa y proteínas. Por lo tanto un conocimiento detallado de la composición y nutrientes de leches no convencionales como las de cabra y búfala podría ser de gran importancia en la nutrición humana. En la presente investigación se realizó una aproximación al comparar la composición de las leches de vaca, cabra y búfala.

En cuanto a su composición se encontró que la leche de búfala presenta mayor porcentaje de grasa, proteína, ST, SNG y lactosa que las leches de cabra y vaca. Estos resultados coinciden con lo reportado por FERNÁNDEZ et al., 2008., quien encontró que la leche de búfala presenta características muy propias que permiten su identificación desde el punto de vista físico-químico, ya que presenta menos agua y más materia seca que la leche de cabra y vaca. Además, los valores de grasa y proteína superan considerablemente a las otras dos especies, lo cual permite apuntar esta especie como una alternativa promisoria en la transformación de varios productos lácteos por su rendimiento en conversión (FERNÁNDEZ et al., 2008).

Respecto al contenido de lactosa se observó que la leche de cabra posee menor contenido comparadas con las leches de vaca y búfala, respectivamente. Dicho resultado concuerda con el estudio reportado por CHACÓN y VILLALOBOS (2005), quienes concluyeron que el contenido de lactosa en la leche de cabra es bajo en comparación con la leche de otras especies animales (aproximadamente de $1 \%$ a $13 \%$ menos que la de vaca y hasta $41 \%$ menos que la humana), lo cual implica que esta leche está asociada a menos problemas de tolerancia a la lactosa (CHACÓN y VILLALOBOS, 2005). De otro lado, hay que resaltar que aunque para las leche de vacas y cabra los valores de grasa y proteína tienden a ser semejantes, se presenta una diferencia en el tamaño de los glóbulos grasos, lo

Tabla 3. Contenidos de Calcio, Fosforo, Potasio y Magnesio en las muestras.

\begin{tabular}{|c|c|c|c|c|c|}
\hline Especie & Muestra & $\mathrm{Ca}(\mathrm{mg} / \mathrm{kg})$ & $P(\mathbf{m g} / \mathrm{kg})$ & $\mathrm{K}(\mathrm{mg} / \mathrm{kg})$ & $M g(\mathbf{m g} / \mathbf{k g})$ \\
\hline \multirow{3}{*}{ Vaca } & 1 & 800 & 581,5 & 1272,5 & 46,3 \\
\hline & 2 & 729,8 & 510 & 1041,6 & 33 \\
\hline & Promedio & $764,9 \pm 49,64$ & $545,75 \pm 50,56$ & $1157,05 \pm 163,27$ & $39,65 \pm 9,40$ \\
\hline \multirow{3}{*}{ Cabra } & 3 & 571,2 & 581,7 & 1134,2 & 42,7 \\
\hline & 4 & 647 & 539,9 & 1151,1 & 47 \\
\hline & Promedio & $609,1 \pm 53,60$ & $560,8 \pm 29,56$ & $1142,65 \pm 11,95$ & $44,85 \pm 3,04$ \\
\hline \multirow{3}{*}{ Búfala } & 5 & 1350,8 & 597,8 & 493,3 & 65,9 \\
\hline & 6 & 982,5 & 672,5 & 671,4 & 66,3 \\
\hline & Promedio & $1166,65 \pm 260,43$ & $635,15 \pm 52,82$ & $582,35 \pm 125,94$ & $66,1 \pm 0,28$ \\
\hline
\end{tabular}


cual hace que la leche de cabra sea más digestible (FERNÁNDEZ et al., 2008; CALVACHE y NAVAS, 2012).

Para el nitrógeno ureico en leche (MUN) se encontraron valores similares entre la leche de búfala y vaca, mientras que la leche de cabra presenta valores muy superiores a las dos anteriores. De acuerdo al estudio realizado por ACOSTA et al., 2005, se pudo observar que para los valores de MUN obtenidos en nuestro estudio la concentración de nitrógeno está muy por encima de los niveles aceptables para este parámetro, el cual oscila entre 9-19 mg/dl de leche. Se puede deducir que en las muestras de leche de cabra analizadas hay excesos de nitrógeno, lo cual podría tener algunos efectos adversos en los procesos de industrialización de los productos lácteos. Lo anterior ha llevado a que una serie de países como Alemania, Suecia, Canadá, USA, entre otros, incorporen su determinación dentro de los análisis rutinarios del control lechero (ACOSTA et al., 2005).

Además, la síntesis de urea en los rumiantes para degradar el exceso de proteína y excretarlo les resta energía para otras funciones como la producción de más leche. De otro lado, los altos niveles de urea en sangre reducen la eficacia del sistema inmunitario del animal, haciéndolo más susceptible a contraer enfermedades (HESS et al., 2011).

Respecto al análisis de ácidos grasos, hay que tener en cuenta que la dieta del animal se refleja en la calidad de su leche. Las variaciones más claras, se dan para el caso de la grasa, que en varias especies de mamíferos suele ser el parámetro más variable de la leche, lo cual implica que la composición puede ser mejorada o empeorada teniendo cuenta la relación ácidos grasos saturados e insaturados en la dieta del animal (ANGULO et al., 2009).

Teniendo en cuenta los resultados anteriores, se puede observar que la leche de cabra es más digestible que la leche de búfala y vaca, ya que presenta mayor cantidad de ácidos grasos de cadena corta y media. Estos ácidos grasos de cadena media (capríco, caproico y caprílico), presentes en mayor cantidad en la leche de cabra, poseen propiedades diferentes a los de cadena larga cuando son metabolizados por el ser humano, especialmente los ácidos caprílico y cáprico, ya que estos ácidos tienen tendencia a proporcionar energía y no a contribuir a la formación de tejido adiposo, además tienen habilidad para limitar y disolver los depósitos de colesterol sérico, lo que se relaciona con una disminución en la incidencia de casos de enfermedades coronarias, fibrosis quística y cálculos biliares (PARK et al., 2007).

De otro lado los ácidos grasos de cadena corta intervienen en el control del peso corporal a través de tres mecanismos principales: estimular la oxidación, disminuir la lipogénesis en tejido adiposo y favorecer la formación de cuerpos cetónicos (KONDYLI et al., 2012), lo cual favorece su consumo.

Adicional a lo anterior, la grasa de la leche caprina no contiene aglutinina, proteína cuya función es agrupar los glóbulos grasos para formar estructuras de mayor tamaño. Por lo tanto, los glóbulos grasos, al estar dispersos, son metabolizados fácilmente por las enzimas digestivas, lo cual favorece a su digestión (KONDYLI et al., 2012).

También se pudo observar que la leche de búfala presento mayor cantidad de ácido palmítico y esteárico comparado con las leches de cabra y vaca respectivamente, lo cual coincide con lo reportado por VERRUMA y SALGADO (2004). Dichos resultados son una ventaja para la salud, dado que los ácidos palmítico y esteárico son considerados ácidos grasos neutros y por lo tanto no afectan los niveles de colesterol en los humanos (SÁYAGO et al., 2008).

Respecto al contenido de minerales, los valores de calcio encontrados en leche de búfala coinciden con lo reportado en el estudio realizado por VERRUMA y SALGADO en el 2004, los cuales reportan que el porcentaje de $\mathrm{Ca}$ en la leche de búfala es mayor que en la de vaca y de cabra (PATIÑO, 2011). El contenido elevado de $\mathrm{Ca}$ en la leche de búfala, le da un valor agregado sobre todo desde el punto de vista nutricional y tecnológico en la elaboración y transformación de productos lácteos (VERRUMA y SALGADO, 2004). 
Además el Ca es uno de los minerales más importantes para el cuerpo humano, ya que ayuda a formar y mantener los dientes y huesos sanos. El Ca también interviene en varias funciones del cuerpo como la coagulación de la sangre, envío y recepción de señales a través del cuerpo, contracción y relajación de músculos, secreción de hormonas y mantenimiento de un ritmo cardiaco normal (BALLARD y MORROW, 2013).

Para el contenido de $\mathrm{P}$ se observa que las leches de las 3 especies analizadas poseen altos contenidos del mineral comparados con la leche humana $(150 \mathrm{mg} / \mathrm{Kg})$; sin embargo la leche búfala posee mayor cantidad del mineral que las leches de cabra y vaca respectivamente, lo cual es de resaltar ya que el $P$ es importante en la nutrición humana porque contribuye junto con las proteínas a la alta capacidad buffer del sistema digestivo (RICHARDSON, 2004), lo cual ayuda en el tratamiento de úlceras gástricas, especialmente cuando la constante irritación causada por la acción de los jugos gástricos provoca daños para el revestimiento del tracto digestivo (RICHARDSON, 2004).

Además, el $P$ es un componente del ácido desoxirribonucleico (ADN) y ácido ribonucleico (ARN), que son esenciales para el crecimiento y diferenciación de las células (CASHMAN, 2006). Como componente de los fosfolípidos, contribuye a la fluidez e integridad de la membrana celular; como fosfato ayuda a mantener el equilibrio osmótico y el balance acido-base, además de que juega un papel esencial en las funciones metabólicas del organismo incluyendo la utilización y transferencia de energía AMP, ADP y ATP. También es importante en la glucogénesis en el transporte de ácidos grasos, en la síntesis de aminoácidos y proteína y en la actividad de la bomba Na/K (CASHMAN, 2006). Cualquier limitación en el suministro de $\mathrm{P}$ se reflejará en un deterioro generalizado de las funciones del cuerpo. En la industria, el $\mathrm{P}$ es muy importante en el incremento y solubilidad de la caseína y actúa sobre la segunda fase de la acción del cuajo (FOX y MCSWEENEY, 1998).

Respecto al contenido de Mg, nuestros resultados coinciden con lo reportado por VERRUMA y SALGADO (2004) y PATIÑO et al., (2011) quienes encontraron en sus estudios que la leche de búfala posee mayor contenido de magnesio que las leches de cabra y vaca. Este macromineral es componente del sistema óseo, de la dentadura y de muchas enzimas. Participa en la transmisión de los impulsos nerviosos, en la contracción y relajación de músculos, en el transporte de oxígeno a nivel tisular y participa activamente en el metabolismo energético (LARSSON et al., 2012).

Además, gran número de estudios han demostrado que el Mg puede beneficiar la presión arterial y ayudar a evitar ataques cardiacos y derrames cerebrales (DEL GOBBO et al., 2013; SONG et al., 2006; XU et al., 2013). Es así como un análisis realizado en el año 2012 por el American Journal of Clinical Nutrition revisó un total de siete estudios que involucraban a más de 240,000 participantes. Los resultados demostraron que la ingesta del magnesio por medio de los alimentos está inversamente relacionada con el riesgo de derrame cerebral isquémico.

Finalmente, en lo que corresponde al tema de minerales, se recomienda tener en cuenta que los niveles de minerales en leche pueden variar de acuerdo con los niveles de minerales en el suelo, el alimento y el forraje que se le suministre al animal. Además, el contenido mineral también puede variar por numerosos factores como la raza, el periodo de lactación, el clima y la estación del año (PARK et al., 2013). Esta puede ser la razón por la cual trabajos realizados en diferentes regiones reportan diferentes niveles de minerales.

\section{Conclusiones}

La leche de búfala presento valores más altos para parámetros tales como la proteína, grasa, lactosa, sólidos totales y sólidos no grasos que las leches de cabra y vaca, lo cual la convierte en una excelente alternativa para la producción y transformación de productos lácteos debido a las cualidades específicas que posee en comparación con las demás. De otro lado la leche de cabra presenta más ácidos grasos esenciales como el linoleico, araquidónico y una proporción mayor de ácidos grasos de cadenas medias y cortas que las leches de vaca y búfala por lo cual es más digestible y saludable para el consumo humano. Respecto a la leche de vaca, a pesar de que no posee las propiedades en composición, ácidos grasos y minerales de las leches de búfala y cabra, esta leche posee niveles importantes de grasa, proteína, minerales y vitaminas importantes en 
la alimentación humana, además de ser el tipo de leche más producido en el mundo, lo que le permitirá seguir siendo una buena competencia para las otras especies de animales productores de leche para el consumo humano.
Agradecimientos: Agradecemos al Comité para el Desarrollo de la Investigación CODI de la Universidad de Antioquia (Proyecto código 8714-2013-5027) y al grupo de investigación en Genética, Mejoramiento y Modelación Animal GaMMA de la Universidad de Antioquia (Sostenibilidad CODI UdeA Código 2014-2015 E01808) por la financiación del proyecto.

\section{Referencias}

ACOSTA, Y. M.; DELUCCHI, M. I.; OLIVERA, M. 2005. Urea en leche: factores que la afectan [serial online]. Sitio Argentino de Producción Animal, 2005. Disponible en: http://www.produccion-animal.com. ar. Consultado: 8-04-2015.

ADLER, S.A.; DAHL, A.V.; JENSEN, S.K. et al. 2013. Fatty acid composition, fat-soluble vitamin concentrations and oxidative stability in bovine milk produced on two pastures with different botanical composition. Livestock Science 154:93-102.

ANGULO, J.; MAHECHA, L.; OLIVERA, M. 2009. Síntesis, composición y modificación de la grasa de la leche bovina: un nutriente valioso para la salud humana. Revista MVZ Córdoba 14 (3):1856-1866.

BALLARD, O.; MORROW, A. L. 2013. Human Milk Composition. Pediatr Clin N Am. 60:49-74.

BARRUCAND, P.; RAYNAL-LJUTOVAC, K. 2007. Variation of whey protein content in goat milk and impact on cheese yield. In: 5th International Symposium on The Challenge to Sheep and Goats Milk Sectors; Alghero, Italy 2007 Abril 18-20. p. 147.

CALVACHE-GARCÍA, I.; NAVAS- PANADERO, A. 2012. Factores que influyen en la composición nutricional de la leche. Revista de ciencias animales 5:73-85.

CASHMAN, K. 2006. Milk minerals (including trace elements) and bone health. International Dairy Journal 16:1389-1398.

CHACÓN-VILLALOBOS, A. 2005. Aspectos nutricionales de la leche de cabra (Capra hircus) y sus variaciones en el proceso agroindustrial. Agronomía Mesoamericana 16 (2):239-252.

COUVREUR, S.; HURTAUD, C.; PEYRAUD J. L. 2006. Milk fat globule size and fatty acid composition variability in dairy cows. Renc. Rech. Rumin 13:301-304.

DEL GOBBO, L.C.; IMAMURA, F.; WU, J.H. et al. 2013. Circulating and dietary magnesium and risk of cardiovascular disease: a systematic review and meta-analysis of prospective studies. Am J Clin Nutr 98 (1):160-173.

DIAN-BO, Z.; YAN-HONG, B.; YUAN-WEN, N. 2015. Composition and characteristics of Chinese Bactrian camel milk. Small Ruminant Research 127: 58-67.

FAOSTAT, 2013. Disponible en: http:// http://www.faostat3.fao.org/browse/Q/QL/S. Consultado: 13-042015. 
FERNÁNDEZ-FARIA, G. H.; DE PAULA-VIEIRA, D. A.; DA SILVA-MACHADO, S. 2008. Comparación de la composición de leche en diferentes especies: una revisión. Simpoets, Cefet-Go 1:104-108.

FOX, P. F.; MCSWEENEY, P. 1998. Dairy Chemistry and Biochemistry. Blackie Academic \& Professional. London.

GUTIÉRREZ, L.F.; MARTÍNEZ, J.C.; BARÓN, M.R. 2010. Contenido de Ácido Linoleico Conjugado (CLA) y Composición de Ácidos Grasos en Algunos Yogures Comerciales de Colombia. Rev.Fac.Nal. Agr.Medellín 63 (2):5685-5692.

GUTIÉRREZ, L.F.; RATTI, C.; BELKACEMI, K. 2008. Effects of drying method on the extraction yields and quality of oils from Quebec sea buckthorn (Hippophae rhamnoides L.) seeds and pulp. Food Chemistry 106 (3):896-904.

HESS, H. D.; FLOREZ, H.; LASCANO, C. E. et al. 2000. Fuentes de variación en la composición de la leche y niveles de úrea en sangre y leche de vacas en sistemas de doble propósito en el trópico bajo de Colombia. Pasturas Tropicales 21 (1):33-42.

INSTITUTO COLOMBIANO DE NORMAS TÉCNICAS Y CERTIFICACIÓN (ICONTEC). NTC 4981. 2001. Alimentos para animales. Determinación del contenido de fosforo. Método espectrofotométrico. Bogotá D.C.

INSTITUTO COLOMBIANO DE NORMAS TÉCNICAS Y CERTIFICACIÓN (ICONTEC). NTC 5151. 2003. Alimento para animales. Determinación de los contenidos de calcio, cobre, hierro, magnesio, manganeso, potasio, sodio y zinc. Método usando espectrometría de absorción atómica. Bogotá D.C.

KANWAL, R.; AHMED, T.; MIRZA, B. 2004. Comparative analysis of quality of milk collected from buffalo, cow, goat and sheep of Rawalpindi/lslamabad region in Pakistan. Asian Journal of Plant Sciences 3 (3):300-305.

KONDYLI, E.; SVARNAS, C.; SAMELIS, J. et al. 2012. Chemical composition and microbiological quality of ewe and goat milk of native Greek breeds. Small Ruminant Research 103 (2):194-199.

LARSSON, S.C.; ORSINI, N.; WOLK, A. 2012. Dietary magnesium intake and risk of stroke: a metaanalysis of prospective studies. American Journal of Clinical Nutrition 95 (2):269-270.

LEITNER, G.; LAVON, Y.; MATZRAFI, Z. et al. 2015. Somatic cell counts, chemical composition and coagulation properties of goat and sheep bulk tank milk. International Dairy Journal (In Press).

MESTAWET, T.A.; GIRMA, A.; DEVOLD, T.G. et al. 2012. Milk production, composition and variation at different lactation stages of four goat breeds in Ethiopia. Small Ruminant Research 105:176-181.

PARK, Y. W.; ALBENZIO, M.; SEVI, A. et al. 2013. Milk Quality Standards and Controls. Milk and Dairy Products in Human Nutrition: Production, Composition and Health:261-287.

PARK, Y. W.; JUAREZ, M.; RAMO, M. et al. 2007. Physico-chemical characteristics of goat and sheep milk. Small Ruminant Research 68:88-113.

PATIÑO, E. M. 2011. Producción y calidad de leche bubalina. Tecnología en marcha 24 (5):25-35. 
PETIT, H.V. 2015. Milk production and composition, milk fatty acid profile, and blood composition of dairy cows fed different proportions of whole flaxseed in the first half of lactation. Animal Feed Science and Technology 205:23-30.

REHMAN, Z.U.; SALARIA, A.M. 2005. Effect of storage conditions on the nutritional quality of UHT processed buffalo milk. J. Chem. Soc. Pak 27 (1):73-76.

RICHARDSON, C.W. 2004. Let's learn about dairy goats and goat's milk. Oklahoma Cooperative Extensión Service. Oklahoma State University. Boletin No 424.

SÁYAGO-AYERDI, S. G.; VAQUERO, M. P.; SCHULTZ-MOREIRA, A. et al. 2008. Utilidad y controversias del consumo de ácidos grasos de cadena media sobre el metabolismo lipoproteico y obesidad. Nutrición Hospitalaria 23 (3):191-202.

SONG, Y.; SESSO, H.D.; MANSON, J.E. et al. 2006. Dietary magnesium intake and risk of incident hypertension among middle-aged and older US women in a 10-year follow-up study. Am J Cardiol 98 (12):1616-1621.

VIEITEZ, I.; IRIGARAY, N.; CALLEJAS, V. et al. 2016. Composition of fatty acids and triglycerides in goat cheeses and study of the triglyceride composition of goat milk and cow milk blends. Journal of Food Composition and Analysis 48:95-101.

VERRUMA, M. R.; SALGADO, J. M. 2004. Análisis químico de la leche de búfala en comparación a la leche de vaca. Scientia Agrìcola. Piracicaba 51 (1):131-137.

XU, T.; SUN, Y.; XU, T. et al 2013. Magnesium intake and cardiovascular disease mortality: A meta-analysis of prospective cohort studies. Int J Cardiol.167 (6):3044-3047.

ZOTOS, A.; BAMPIDIS, V.A. 2014. Milk fat quality of Greek buffalo (Bubalus bubalis). Journal of food composition and Analysis 33 (2):181-186. 